\title{
Chromosomal localization of the major satellite DNA family (FA-SAT) in the domestic cat
}

\author{
S. Santos, R. Chaves, and H. Guedes-Pinto \\ Department of Genetics and Biotechnology, Centre of Genetics and Biotechnology of the University of Trás-os-Montes and Alto Douro - CGB/UTAD, Vila Real (Portugal)
}

\begin{abstract}
.
A major satellite DNA sequence was isolated from the cat genome and its sequencing data revealed homology to the FA-SAT family. In situ hybridization of the cat satellite DNA and telomeric sequences to cat chromosomes, together with staining of constitutive heterochromatin, allowed the physical mapping of the FA-SAT sequences, and also an overall constitutive heterochromatin study in cat chromosomes.
\end{abstract}

\section{Introduction}

The karyotype of Felis catus consists of 38 chromosomes. Comparative genomic studies have shown that the cat karyotype is remarkably conserved and closely resembles the putative ancestral mammalian karyotype (Wienberg et al., 1997; Murphy et al., 2001). Furthermore, the proposed $2 \mathrm{n}=42$ ancestral carnivore karyotype is comparable to that of the cat (Murphy et al., 2001).

Constitutive heterochromatin is known to contain tandemly repeated satellite DNAs. Analysis of these repeats suggests that the sequences are rapidly evolving, and hence they are valuable evolutionary markers. Fanning (1987) reported the origin and evolution of a major feline satellite DNA. Fanning et al. (1988) and Modi et al. (1988) published the earliest and most unique comparative studies regarding carnivore satellite DNA. Modi et al. (1988) found satellite DNA sequences primarily at telomeres, but also at centromeres in the several species of felids examined. Domestic cat satellite DNA sequences were detected mostly on cat chromosomes belonging to the $\mathrm{D}, \mathrm{E}$ and $\mathrm{F}$ groups and the $\mathrm{X}$ chromosome.

In the present work, we isolated and cloned a major satellite DNA family from the domestic cat genome. One of the clones was sequenced and showed homology to the FA-SAT cat satellite DNA family. This repeat was mapped to cat chromosomes using fluorescence in situ hybridization (FISH). In order to localize precisely the cat FA-SAT family to specific chromosome regions, we also examined the constitutive heterochromatin with $\mathrm{C}$ banding techniques, and the telomere regions by in situ hybridization using a vertebrate telomeric sequence.

\section{Material and methods}

\section{Felis catus satellite DNA isolation and sequence analysis}

Cat genomic DNA was isolated from tissue samples (muscle) using standard methods. Cat satellite DNA sequences were isolated from cat genomic DNA digested with BamHI and separated by gel electrophoresis. A prominent 500-bp fragment was cut from the gel and cloned in the BamHI site of pZErO-1 (Invitrogen Life Technology). One clone was selected for further analysis and the nucleotide sequences were determined in both strands by automatic sequencing. DNA sequences were analyzed using BLASTN searches and EMBL databases. Sequence data from the clone analyzed was already deposited in the GenBank database (accession number AY425348)

\section{Chromosome preparations}

We analyzed 22 domestic cats in which five individuals were males and the remaining 17 were females. The cat chromosome preparations were made from short-term lymphocyte cultures of whole blood and from fibroblast cell cultures using standard procedures

\section{GTG and GTD-banding}

Air-dried slides were aged at $65^{\circ} \mathrm{C}$ for $5 \mathrm{~h}$ or overnight, and then were submitted to standard procedures of G-banding with trypsin. Some chromosome preparations were stained at this stage with Giemsa, observed and then subjected to subsequent C-banding. Other chromosome preparations were used for subsequent FISH and were not stained at this stage. These latter preparations were fixed with paraformaldehyde as published before (Chaves et al., 2004). Inversion of the DAPI color in Adobe Photoshop (version 5.0) revealed Gbanding of chromosomes (GTD-banding, G-bands by trypsin with DAPI) allowing their identification. The organization of the karyotypes was done according to the previous cat karyotype standardization of Cho et al. (1997).

\section{CBP-banding following G-bands}

After G-banding or fluorescent in situ hybridization procedures, slides were destained and subjected to C-banding. CBP-banding (Cbands by barium hydroxide using propidium iodide) was done as described by Chaves et al. (2004).

\section{Probe preparation and fluorescence in situ hybridization (FISH)}

Metaphase chromosomes were hybridized with the cat satellite DNA sequences and telomeric probes after PCR labeling with biotin16-dUTP (Sigma) or digoxigenin-11-dUTP (Roche). In situ hybridization procedures were done using standard procedures. Biotin-labeled probes were detected with avidin conjugated to fluorescein isothiocyanate (FITC) (Vector Laboratories); digoxigeninlabeled probes with 5-carboxy-tetramethylrhodamine (5-TAMRA) conjugated to anti-digoxigenin (Roche). 


\section{Chromosome observation}

Chromosomes were observed with a Zeiss Axioplan 2 Imaging microscope coupled to Axiocam digital camera and AxioVision software (version2.0.5; Zeiss). For each individual tested, at least 30 metaphases were analyzed (in each technical procedure performed).

\section{Results and discussion}

A BamHI fragment of approximately $500 \mathrm{bp}$, corresponding to a prominent band in an ethidium bromide-stained gel was cloned from cat genomic DNA. One clone was sequenced and a search of GenBank identified it as being homologous (with $98 \%$ sequence similarity) to the cat satellite DNA family FA-SAT reported by Fanning (1987) with accession number X06372. The clone from the FA-SAT DNA family was hybridized to Southern blots of restriction digested genomic DNA from cat (data not shown) and a hybridization ladder pattern characteristic of cat satellite sequences was observed as previously demonstrated by Fanning et al. (1988) and Modi et al. (1988).

In situ hybridization of the cat satellite DNA and telomeric sequences to cat chromosomes, together with the staining of constitutive heterochromatin $(\mathrm{CH})$ by $\mathrm{C}$-banding techniques, allowed the physical mapping of the cat satellite DNA sequences, and also an overall constitutive heterochromatin study in cat chromosomes (Figs. 1 and 2).

Figure 1a shows hybridization of the cat satellite probe to cat chromosome preparations. Most of the satellite DNA signals map to telomeric regions, and are clearly not present in all chromosomes. Chromosomes E1, F1 and the $\mathrm{X}$ are easily identified since they demonstrate a characteristic in situ hybridization pattern (Fig. 1a). Subsequent C-banding procedures (Fig. 1b) were done in order to colocalize the satellite DNA sequences to the $\mathrm{CH}$ regions. In a general analysis, the cat satellite DNA sequences are co-localized with the Cbands (see Fig. 1a, b). In Fig. 1c, d, we present the two-color in situ hybridization with the telomeric probe only (Fig. 1c), and with telomeric and cat satellite DNA sequences, in order to verify the telomeric localization of the satellite DNA sequences. Most of the satellite DNA sequences co-localized with the telomeric probe (Fig. 1d). In Fig. 1, the two types of FISH patterns found in cat $X$ chromosomes can be observed: in one type (Fig. 1a), we found both telomeric regions in situ hybridized with satellite DNA; in the other, only the telomeric $\mathrm{X}$ chromosome short arm demonstrated satellite DNA sequences (Fig. 1d). This $X$ chromosome polymorphism was the only specific inter-individual variation found in the 22 individuals analyzed, with $75 \%$ of the $\mathrm{X}$ chromosomes showing signals at both telomeric regions after in situ hybridization of the FA-SAT sequences.

Figure 2 summarizes the most representative results from GTD, $\mathrm{CBP}$ and FISH analysis. The FA-SAT family was never detected in cat chromosomes A1, A2, B1, B4, C1, C2 and D3. Some cat chromosomes showed satellite DNA sequences consistently polymorphic between homologous chromosome pairs in all animals tested (e.g. chromosomes A3, B3, D2, D4, E1, E2 and F2). Even so, the satellite DNA sequences could be found in telomeric regions of both $\mathrm{p}$ and $\mathrm{q}$ arms of chromosomes A3, B3, D2, E1, E2, E3 and X; only in one of the telomeric regions of chromosomes B2, D1 and D4; in both telomeric and centromeric regions of chromosomes F1 and F2; only in pericentromeric region of chromosome $\mathrm{Y}$; finally, chromosome F1 (band q1.13) also demonstrated a large interstitial block of satellite DNA. All chromosomes presented a hybridization signal of the vertebrate telomeric sequences in the telomere regions of both arms (Fig. 2). Some telomeric signals showed to be consistently polymorphic between homologous chromosome pairs and chromosome $\mathrm{F} 1$ also demonstrated a polymorphic telomeric signal in an interstitial region (band q1.13). The overall analysis of Fig. 2 shows that not all $\mathrm{CH}$ blocks presented strong signals of the FA-SAT family (e.g. chromosomes D2 or D4 pericentromeric C-bands and chromosome E1 interstitial C-band), meaning that the FA-SAT family is only one of the $\mathrm{CH}$ components. Moreover, our results are in agreement with those from Modi et al. (1988) concerning the primary chromosomal localization of FA-SAT family (telomeric regions). Yet, Modi et al. (1988) did not report the presence of FA-SAT family to individual chromosomes (e.g. chromosomes B2 and Y), neither to chromosome regions.

The presence of this cat satellite DNA family in the $Y$ chromosome, and simultaneously in some of the autosomes, is a very interesting and uncommon feature. Similar cases are reported in other species, as for instance the presence of the repeated sequences in the human Y chromosome and some of the autosomes (Cooper et al., 1992). Comparative mapping strongly demonstrates that sex chromosome differentiation has occurred independently (Graves and Shetty, 2000). Besides, occurrence of the same satellite DNA in autosomes and sex chromosomes has been considered a primitive condition for the sex chromosomes (Gallagher et al., 1999). Moreover, in contrast to the overall placental mammalian $\mathrm{Y}$ chromosomes that are predominantly or completely heterochromatic (Graves and Shetty, 2000), the cat Y chromosome (see Fig. 2) is not entirely stained by C-banding, what suggests a lesser degree of degradation compared to the general mammalian $\mathrm{Y}$ chromosomes. Thus, our results suggest that the cat $\mathrm{Y}$ (and also the $\mathrm{X}$ ) chromosome could be considered in a primitive condition and therefore represents a conserved form.

\section{Acknowledgements}

We thank the Veterinary Hospital of Trás-os-Montes, Vila Real, Portugal, for the tissue samples.

\section{References}

Chaves R, Santos S, Guedes-Pinto H: Comparative analysis (Hippotragini versus Caprini, Bovidae) of $\mathrm{X}$ chromosome's constitutive heterochromatin by in situ restriction endonuclease digestion: $\mathrm{X}$ chromosome constitutive heterochromatin evolution. Genetica, in press (2004)

Cho K-W, Youn H-Y, Watari T, Tsujimoto H, Hasegawa A, Satoh H: A proposed nomenclature of the domestic cat karyotype. Cytogenet Cell Genet 79:71-78 (1997).

Cooper KF, Fisher RB, Tyler-Smith C: Structure of the pericentric long arm region of the human Y chromosome. J Mol Biol 228:421-432 (1992).

Fanning T: Origin and evolution of a major feline satellite DNA. J Mol Biol 197:627-634 (1987).Fanning TJ, Modi WS, Wayne RW, O'Brien SJ: Evolution of heterochromatin-associated satellite DNA loci in felids and canids (Carnivora). Cytogenet Cell Genet 48:214-219 (1988).

Gallagher DS, Davis SK, Donato M, Burzlaff JD, Womack JE, Taylor JF, Kumamoto AT: A molecular cytogenetic analysis of the tribe Bovini (Artiodac tyla: Bovidae: Bovinae) with an emphasis on sex chromosome morphology and NOR distribution. Chromosome Res 7:481-492 (1999).

Graves JAM, Shetty S: Comparative genomics of vertebrates and the evolution of sex chromosomes, in Clark M (ed): Comparative Genomics, pp 153-205 (Kluwer Academic Publishers, Massachusetts 2000).

Modi WS, Fanning TG, Wayne RK, O'Brien SJ: Chromosomal localization of satellite DNA sequences among 22 species of felids and canids (Carnivora). Cytogenet Cell Genet 48:208-213 (1988).

Murphy WJ, Stanyon R, O'Brien SJ: Evolution of mammalian genome organization inferred from comparative gene mapping. Genome Biol 2:0005.1-0005.8 (2001).

Wienberg J, Stanyon R, Nash WG, O’Brien PCM, Yang F, O’Brien SJ, FergusonSmith MA: Conservation of human vs. feline genome organization revealed by reciprocal chromosome painting. Cytogenet Cell Genet 77:211-217 (1997). 

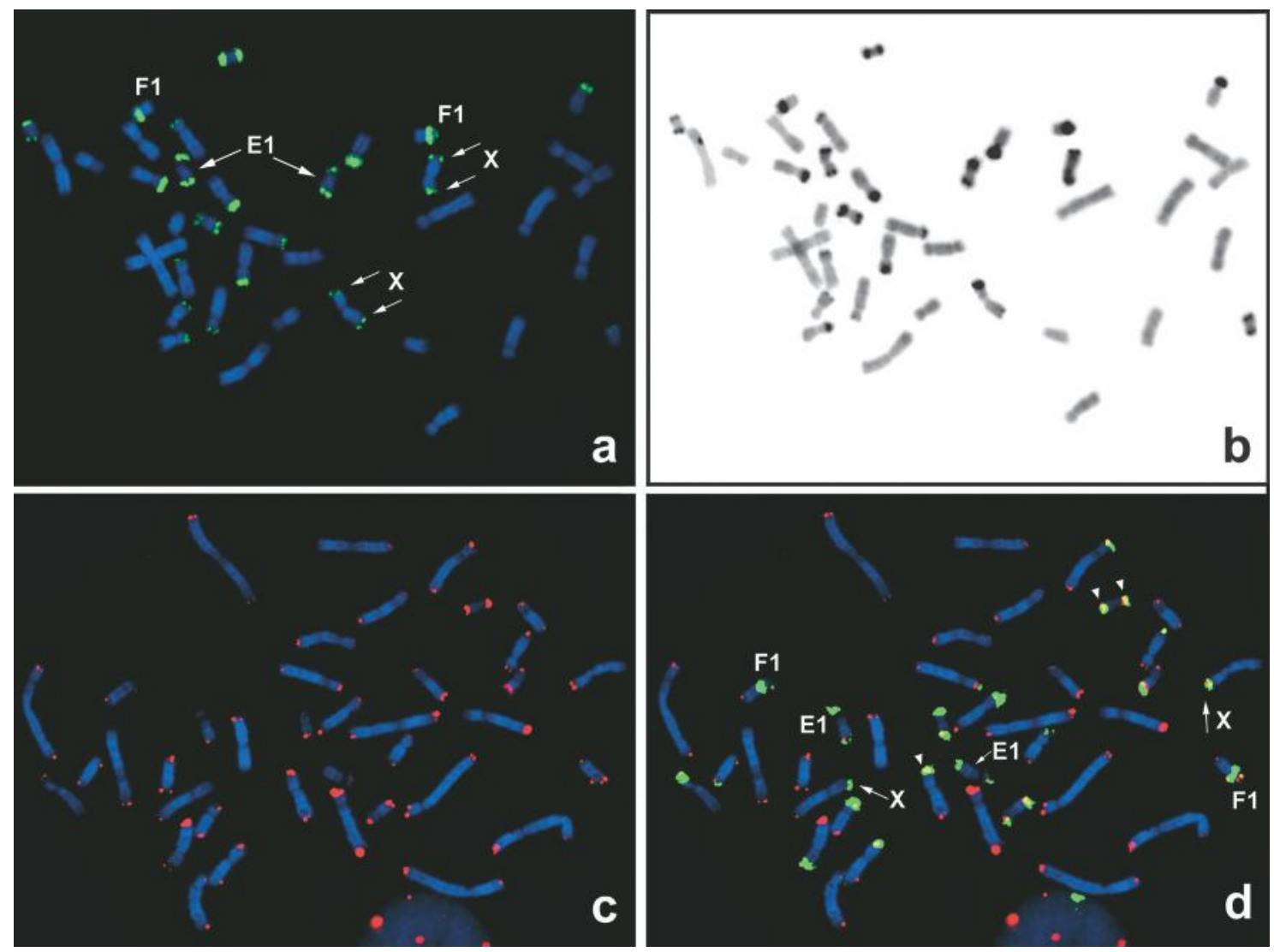

Fig. 1. (a) In situ hybridization of the FA-SAT family (green signal) to cat chromosome preparations (counterstained blue with DAPI). Chromosomes E1, F1 and X are easily identified by their characteristic in situ hybridization pattern. (b) Sequential C- banding of the same metaphase. (c, d) Simultaneous in situ hybridization of the FA-SAT (green signal) and telomere (red signal) probes. In c, only hybridization of the telomere probe is shown.

\begin{tabular}{|c|c|c|c|c|c|c|c|c|c|c|c|c|c|c|c|c|c|}
\hline & & & \multicolumn{3}{|c|}{ GTD + FISH } & & & & \multicolumn{3}{|c|}{ GTD + FISH } & & & & \multicolumn{3}{|c|}{ GTD + FISH } \\
\hline & GTD & CBP & FA-SAT & Tel. & \begin{tabular}{|c|c|} 
FA-SAT \\
+ Tel. \\
\end{tabular} & & GTD & CBP & FA-SAT & Tel. & \begin{tabular}{|c|} 
FA-SAT \\
+ Tel. \\
\end{tabular} & & GTD & CBP & FA-SAT & Tel. & $\begin{array}{c}\text { FA-SAT } \\
\text { + Tel. } \\
\end{array}$ \\
\hline \multirow{2}{*}{ A1 } & F & & & & & |B4 & 83 & & & & & E1 & ริะี & 88 & $\therefore$ & 8. & A \\
\hline & 8 & f & & & & & & & & & & E2 & 86 & 80 & $\because$ & $i$ & d8 \\
\hline \multirow[t]{2}{*}{ A2 } & & & & & & C1 & & & & & & E3 & ะ8 & 88 & $\because$ & $\therefore$ & है? \\
\hline & & & & \multirow[b]{2}{*}{ है } & & & & & & & & F1 & 98 & 88 & 80 & 8 & है 1 \\
\hline A3 & 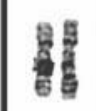 & ํㅐ을 & 8 & & 8 & C2 & 88 & & 8 & 81 & & F2 & 90 & 06 & d & 10 & 11 \\
\hline \multirow[t]{2}{*}{ B1 } & \multirow{2}{*}{ 量 } & \multirow{2}{*}{8} & & & \multirow{2}{*}{11} & |D1 & 8 & 8 & 18 & & & $\mathbf{X X}$ & 8 & & & & \\
\hline & & & & & & D2 & ถ้สี้ & 题 & 16 & & & \multirow[t]{2}{*}{$\mathbf{X Y}$} & 80 & & $8^{\circ}$ & 80 & \\
\hline B2 & 量 & 80 & & & 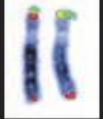 & D3 & 8 & 80 & 14 & & 12 & & & & & & \\
\hline B3 & 8 & 88 & & & & D4 & 能 & 38 & 80 & (I) & है & & & & & & \\
\hline
\end{tabular}

Fig. 2. Most representative results of the GTD, CBP and FISH analyses for each cat chromosome. All chromosomes were pre-identified by Gbanding. 